Proceedings of Anticancer Research

Review Article

\title{
Potential Value of Splenectomy for the Life Quality and Long-term Survival of Patients with Cirrhosis
}

Tuanmao Guo ${ }^{1 \#}$, Maicang Gao ${ }^{2 \#}$, Yanli Xing ${ }^{1}$, Xi-Min Qiao ${ }^{1 *}$

${ }^{1}$ Department of Surgery, Xianyang Central Hospital, Xianyang 712000, China;

${ }^{2}$ Department of Intensive Care Unit, the First Affiliated Hospital, Shaanxi College of Traditional Chinese Medicine, Xianyang 712000 , China

${ }^{\#}$ These authors are regarded as the co-first authors

\begin{abstract}
Patients with cirrhosis will gradually develop portal hypertension (PHT) and esophagogastric varices, and subsequently the more severe and often lethal complications such as bleeding esophageal varices, ascites, spontaneous bacterial peritonitis, renal dysfunction and hepatic encephalopathy. To date, there is still not any effective therapy for the prevention of the development these serious complications. The initial factor leading to PHT is an increase in hepatic resistance. Then, the increase in portal blood flow contributes to the maintenance and exacerbation of PHT, as well as the formation of esophagogastric varices and lethal complications. Increasing evidence shows that, enlarged spleen not only contributes to the increase in portal venous inflow, but also plays a contributory role in promoting almost all lethal complications of cirrhosis through its resulted hypersplenism together with other mechanisms. So, we hypothesize that prophylactic splenectomy may efficiently delay the progression of cirrhosis and improve life quality and long-term survival of patients with cirrhosis.
\end{abstract}

Publication date: September, 2020

Publication online: 30 September, 2020

*Corresponding author: Xi-Min Qiao,gtm0619@163. com

\section{Introduction}

All patients with cirrhosis will eventually develop portal hypertension and esophagogastric varices, and subsequently more severe and often lethal complications such as bleeding esophageal varices, ascites, spontaneous bacterial peritonitis, renal dysfunction and hepatic encephalopathy. It has been suggested in a quantity of studies that most of these complications might be somewhat related with spleen.

\subsection{Portal hypertension}

The initial factor leading to portal hypertension (PHT) is an increase in hepatic resistance. Later, an increase in portal blood flow contributes to maintaining and exacerbating many of the complications of cirrhosis, especially when portal-systemic collaterals are extensive $\mathrm{e}^{[1]}$. The mechanisms underlying this splanchnic hyperemia are not fully understood. According to Ohm's law, portal venous pressure $(\mathrm{P})$ is the product of vascular resistance (R) and blood flow (Q) in the portal bed $(\mathrm{P}=\mathrm{Q} \times \mathrm{R})$. Q here is composed of superior mesenteric venous flow and splenic venous flow. The increase in the splenic venous blood flow in patients with cirrhosis contributes a lot to portal venous pressure. PHT can promote the formation and development of splanchnic hyperdynamic circulation and splanchnic vasculapathy, making the vessels unable to constrict and the blood flow not regulated by elastic membrane, which in turn benefits the development of hyperdynamic circulation ${ }^{[2]}$.

\subsection{Variceal bleeding}

In liver cirrhosis, variceal bleeding is the last in a chain of events initiated by the increase in portal venous pressure. Once varices have formed, they tend to increase in size and eventually to bleed. When hepatic venous pressure gradient goes above $10 \mathrm{mmHg}$ the patient is at risk of developing varices, and when hepatic venous pressure gradient reaches $12 \mathrm{mmHg}$ variceal bleeding might occur ${ }^{[3,4]}$. Bleeding occurs in 30\%-40\% 
of cirrhotic patients once varices have formed. The first episode of variceal bleeding is associated with mortality between $17 \%-57 \%{ }^{[5]}$, and approximately two thirds of the survivors who do not receive active treatment might suffer from recurrent episode of hemorrhage with a high risk of death of about $40 \%$ to $50 \%{ }^{[4]}$. Factors related to the risk of variceal bleeding include portal venous pressure, endoscopic features of varices and the location of varices ${ }^{[4-6]}$.

\subsection{Hypersplenism}

Splenic sequestration and destruction of platelets, white blood cells and red blood cells in the portal hypertension-induced enlarged spleen is defined as hypersplenism ${ }^{[7]}$. Enlargement of the spleen is one of the most critical spleen problems which may affect the function of this organ in the immune system, making the risk of developing infections to a higher level. This is due to the fact that when the organ enlarges in its size, it tends to trap more than required red and white blood cells and platelets at an abnormal rate, which causes a lack of the amount of blood cells in the blood stream which are required to protect the body against infections. The more the organ traps the blood cells, the more it increases in size and eventually causes thrombocytopenia, hypoleukemia and anemia ${ }^{[8]}$.

\subsection{Bacterial infection}

The most frequent infective complications of cirrhosis include spontaneous bacterial peritonitis (SBP), urinary tract infections, respiratory infections, and bacteremia, mostly due to the concomitant presence of various facilitating mechanisms such as changes in the reticuloendothelial system, decreased opsonic activity of the ascitic fluid, neutrophil leukocyte dysfunction, and iatrogenic factors ${ }^{[9]}$. Among these infections, SBP occurs in approximately $8 \%$ of cirrhosis patients with ascites. It carries a mortality of $25 \%$ and recurs in $70 \%$ of patients within a year ${ }^{[10]}$. Recently, bacterial infections and/or endotoxaemia have been associated with failure to control variceal bleeding, more early variceal rebleeding, abnormalities in coagulation, vasodilatation of the systemic vasculature, and worsening liver function ${ }^{[11,12]}$. It is widely considered that the incurrence and development of bacterial infection is related with hypersplenism.

\subsection{Hepatorenal syndrome}

Hepatorenal syndrome (HRS) is a common problem in patients with advanced cirrhosis and ascites. The annual incidence of HRS in cirrhosis patients has been estimated as $8 \%$. This syndrome may develop spontaneously or be precipitated by factors that induce renal hypoperfusion. Bacterial infections, especially $\mathrm{SBP}$, are by far the most frequent precipitating causes of HRS.

\section{Hypothesis}

In view of the potential contributory role of spleen in formation and development of the complications of cirrhosis, we put forward the hypothesis that prophylactic splenectomy might improve life quality and long-term survival of the patients with cirrhosis.

\section{Discussion}

Bleeding from ruptured esophagogastric varices, which is initiated by the increase in portal venous pressure, is the most severe complication of cirrhosis and the cause of death in about one third of patients. During the last decade, management of variceal hemorrhage has been well developed.

For primary prophylaxis, non-selective $\beta$-blockers have remained the cornerstone in the treatment for high portal venous tension. However, a long-term decrease in portal pressure is achieved only in $30 \%-40 \%$ of patients, whereas an additionally estimated $10 \%-20 \%$ of patients discontinue $\beta$-blockers because of intolerance ${ }^{[13]}$.

To control acute variceal bleeding, treatment modalities such as vasoconstrictors, balloon tamponade, endoscopic injection sclerotherapy (EIS), transjugular intra hepatic portasystemic (TIPS) and endoscopic variceal ligation (EVL) may be employed. These techniques can achieve variceal obliteration (sometimes called "eradication"). However, gastroesophageal varices will eventually recur. Once acute bleeding is successfully controlled, rebleeding may occur in approximately two thirds of patients if further preventive measures are not taken $^{[14]}$. Meta-analysis of randomized controlled trials indicates that banding seems to be more effective than $\beta$-blockers at preventing a first bleeding episode, but this does not translate to improved survival ${ }^{[15]}$. In the prevention of recurrent variceal bleeding, TIPS is found to be able to reduce the rebleeding rate effectively but increase hepatic encephalopathy without improving survival $^{[16]}$.

In brief, there is not any effective therapy for the prevention of the development of varices and variceal bleeding currently. As a consequence, the search for potential new strategies is needed. But it might be necessary to re-evaluate the existing managements of variceal hemorrhage before further investigation. Compared to the local treatments (EIS, EVL, left gastric artery embolization), the managements including all 
kinds of shunts (splenorenal shunts, portacaval shunt, TIPS), partial splenic embolization and splenectomy do not aim at strengthening local bleeding cite or cutting down the blood supply of the local cite, but alleviate the hypertension in portal veins, which in turn significantly reduces the rebleeding rate.

Among the existing managements of variceal hemorrhage, splenectomy may be the most promising one. The blood flow is increased in the superior mesenteric arteries and splenic arteries of patients with cirrhosis and portal hypertension, which in turn increases the portal blood flow, exclusively in patients with splenomegaly ${ }^{[17]}$. PHT, partly due to increased portal blood flow, is responsible for the severe and often lethal complications of cirrhosis, such as bleeding esophageal varices, ascites, renal dysfunction and hepatic encephalopathy. A hepatic venous pressure gradient (HVPG) over $10 \mathrm{mmHg}$ is a strong predictor for the development of varies severe complications of cirrhosis varices. Besides, a study has found that for every $1 \mathrm{mmHg}$ increase in HVPG there is an $11 \%$ increase in the risk of clinical decompensation ${ }^{[18]}$. Over the years, it has become clear that a decrease in portal pressure is not only protective against the risk of variceal (re)bleeding but also associated with a lower long-term risk of developing other complications of PHT (hepatic encephalopathy, spontaneous bacterial peritonitis, hepatorenal syndrome) and an improved long-term survival ${ }^{[19]}$. Only if HVPG is substantially reduced below $12 \mathrm{~mm} \mathrm{Hg}$ or by more than $20 \%$ of baseline levels, there is a marked reduction in the risk of bleeding ${ }^{[20,21]}$. While after splenectomy, the portal blood flow may be significantly reduced. It has been reported that the flow is reduced by $27 \%$ in the recent postoperatory period and by up to $37 \%$ in the first to the second year after surgery, and remains stable after this period, and despite the reduction of portal flow in response to surgery, there is no deterioration of hepatic function ${ }^{[22]}$. From this sense, splenectomy may be superior to splenorenal shunts, portacaval shunt, TIPS, partial splenic embolization as a supportive intervention for cirrhotic patients with PHT and hypersplenism. More importantly, splenectomy may promptly correct thrombocytopenia, hypoleukemia and anemia, which contributes to the improvement of life quality and longterm survival of the patients with cirrhosis, and it may also diminish virus burden in cirrhotic patients with $\mathrm{HCV}$ infection at least in part, through augmentation of natural killer cell activity ${ }^{[23]}$.

Aside from its abnormal sequestration and destruction of blood cells and its contribution to increasing portal blood flow, the spleen may be a source of a vasoactive factor, playing an important role in cardiovascular regulation. The splenorenal reflex-mediated reduction in renal vascular conductance exacerbates sodium and water retention in the kidneys and may eventually contribute to renal dysfunction ${ }^{[24]}$. And splenectomy could abrogate these fuctions. Meanwhile, splenectomy in general does not cause any severe postoperative complications, which suggests the existence of strong compensatory mechanisms for the loss of the various functions of the spleen.

The current timing of splenectomy might also need reconsideration. Though $\mathrm{PHT}$ is a severe, almost unavoidable complication of chronic liver diseases, none the less splenectomy almost always definitely benefits the patients no matter how late it is performed. However, as growing evidence shows that spleen contributes a lot to maintaining and exacerbating many of the serious complications of cirrhosis with portal hypertension, early splenectomy might be more beneficial in improving life quality and long-term survival of the patients.

However, it should also be noted that the longterm effects of splenectomy, in terms of improved hematological, hepatic function and immunologic status, are still not guaranteed by sound evidence. Furthermore, how early should splenectomy be performed is a crucial question. Further research is required on the timing of splenectomy and long-term survival of the patients with cirrhosis post splenectomy.

Conflicts of interest statement

None declared.

\section{References}

[1] Poordad FF. Presentation and complications associated with cirrhosis of the liver[J]. Curr Med Res Opin. 2015;31(5):925-37. doi: 10.1185/03007995.2015.1021905.

[2] Zarzaur BL, Dunn JA, Leininger B, et al. Natural history of splenic vascular abnormalities after blunt injury: A Western Trauma Association multicenter trial[J]. J Trauma Acute Care Surg. 2017;83(6):999-1005. doi: 10.1097/ TA.0000000000001597.

[3] Villanueva C, Graupera I, Aracil C, et al. A randomized trial to assess whether portal pressure guided therapy to prevent varicealrebleeding improves survival in cirrhosis[J]. Hepatology, 2017;65(5):1693-1707. doi: 10.1002/hep.29056.

[4] Habib A, Sanyal AJ. Acute variceal hemorrhage[J]. Gastrointest Endosc Clin N Am. 2007;17:223-52.

[5] Zaman A. Current Management of Esophageal Varices[J]. Curr Treat Options Gastroenterol. 2003;6:499-507.

[6] Vilaseca M, Guixé-Muntet S, Fernández-Iglesias A, et al. Advances in therapeutic options for portal hypertension[J]. Therap Adv Gastroenterol. 2018;11:1756284818811294. doi: $10.1177 / 1756284818811294$.

[7] Wang YB, Zhang JY, Zhang F, et al. Partial Splenic Artery Embolization to Treat Hypersplenism Secondary to Hepatic Cirrho- 
sis: A Meta-Analysis[J]. Am Surg. 2017;83(3):274-283.

[8] Lv Y, Yee Lau W, Wu H, et al. Causes of peripheral cytopenia in hepatitic cirrhosis and portal hypertensive splenomegaly[J]. Exp Biol Med (Maywood). 2017;242(7):744-749. doi: 10.1177/1535370217693113.

[9] Yang YY, Lin HC. Bacterial infections in patients with cirrhosis[J]. J Chin Med Assoc. 2005;68:447-51.

[10] Castellote J, López C, Gornals J, et al. Rapid diagnosis of spontaneous bacterial peritonitis by use of reagent strips[J]. Hepatology. 2003; 37:893-6.

[11] Piano S, Morando F, Carretta G, et al. Predictors of Early Readmission in Patients With Cirrhosis After the Resolution of Bacterial Infections[J]. Am J Gastroenterol. 2017;112(10):15751583. doi: 10.1038/ajg.2017.253.

[12] Basili S, Ferro D, Violi F. Endotoxaemia, hyperfibrinolysis, and bleeding in cirrhosis[J]. Lancet. 1999;353:1102.

[13] Laleman W, Van Landeghem L, Wilmer A, et al. Portal Hypertension: From Pathophysiology to Clinical Practice[J]. Liver International. 2005;25:1079-90.

[14] Abraldes JG, Villanueva C, Aracil C, et al. Addition of Simvastatin to Standard Therapy for the Prevention of Variceal Rebleeding Does Not Reduce Rebleeding but Increases Survival in Patients With Cirrhosis[J]. Gastroenterology. 2016;150(5):1160-1170.e3. doi: 10.1053/ j.gastro.2016.01.004.

[15] Tripathi D, Graham C, Hayes PC. Variceal band ligation versus beta-blockers for primary prevention of variceal bleeding: a meta-analysis[J]. Eur J Gastroenterol Hepatol. 2007;19:83545.

[16] Escorsell A, Bañares R, García-Pagán JC, et al. TIPS versus drug therapy in preventing variceal rebleeding in advanced cirrhosis: a randomized controlled trial[J]. Hepatology. 2002; 35:385-92.

[17] Iwakiri Y. Pathophysiology of portal hypertension[J]. Clin Liver Dis. 2014;18(2):281-91. doi: 10.1016/j.cld.2013.12.001.

[18] Afdhal N, Everson GT, Calleja JL, et al. Effect of viral suppression on hepatic venous pressure gradient in hepatitis $\mathrm{C}$ with cirrhosis and portal hypertension[J]. J Viral Hepat. 2017;24(10):823-831. doi: 10.1111/jvh.12706.

[19] Laleman W, Nevens F. Cirrhotic portal hypertension: current and future medical therapy for primary and secondary prevention of variceal bleeding[J]. Minerva Med. 2006; 97: 32545.

[20] Brunner F, Berzigotti A, Bosch J. Prevention and treatment of variceal haemorrhage in 2017[J]. Liver Int. 2017;37 Suppl 1:104-115. doi: 10.1111/liv.13277.

[21] Feu F, Garcia-Pagan JC, Bosch J, et al. Relation between portal pressure response to pharmacotherapy and risk of recurrent variceal haemorrhage in patientswith cirrhosis[J]. Lancet. 1995; 8982:1056-9.

[22] Carvalho DL, Capua A Jr, Leme PL. Portal flow and hepatic function after splenectomy and esophagogastric devascularization[J]. Int Surg. 2008;93:314-20.

[23] Feng B, Zhang W, Luo BF, et al. Effect of spleen operation on antiviral treatment in hepatitis $\mathrm{C}$ virus-related cirrhotic patients[J]. World J Gastroenterol. 2014;20(41):15387-97. doi: 10.3748/wjg.v20.i41.15387.

[24] Hamza SM, Kaufman S. Role of spleen in integrated control of splanchnic vascular tone: physiology and pathophysiology[J]. Can J Physiol Pharmacol. 2009; 87:1-7. 